\title{
Index for Volume 19 of Molecular Plant-Microbe Interactions
}

\section{AUTHOR AND SUBJECT INDEX}

Abe, M., 441

Acosta, J., 1385

Agrobacterium spp.

-A. rhizogenes, on Phaseolus spp., transformation, functional genomics, 1385

-A. tumefaciens, on Arabidopsis thaliana, transcriptome, 665

Aikawa, T., 280

Alamillo, J. M., 350, 541

Alfalfa (see also Medicago spp.)

—nodulation: metabolite profiles, physiological adaptations, 998; transcriptomic dissection, 363

Alfano, J. R., 1167, 1193

Allen, C., 69

Allen, R., 463

Alnus firma, hemoglobin gene, nitric oxide detoxification, 441

Alternaria spp.

-A. alternata, $\mathrm{G}$ protein signaling, pathogenesis, 1280

-A. brassicicola, functional genomics, gene disruption method, 7

Alvarado-Affantranger, X., 1385

Alvarez, A., 69

Alvarez, M. E., 577

Amiar, Z., 550

Amiour, N., 988

Anderson, A. J., 924

Ando, S., 80

Apple, fire blight: pathogenicity effector, 53, 359; pathogenicity and type III effector, 16

Arabidopsis: black spot, gene disruption method, genomics, 7; Colletotrichum spp., nonhost resistance, actin filament function, 270; Plasmodiophora brassicae, metabolism, hormone and proteome approach, 1431; Pseudomonas syringae, pathway regulation, $h r p L$ and $h r p R S, 976$; Pseudomonas syringae, profiling, $\mathrm{HrpL}$ regulon, coregulated genes, 1167;

Pseudomonas syringae, type III secretion system effector proteins, 1180 ;

Pseudomonas syringae and Botrytis cinerea, resistance and salicylic acid, yeast effect, 1138; Verticillium longisporum pathosystem, signals, cytosolic NPT 1 and RFO1, 958

-A. thaliana: Agrobacterium tumefaciens response, transcriptome, 665; Fusarium spp., trichothecenes and elicitors, 512; gene enhancing susceptibility to Botrytis cinerea, 931; jasmonate-insensitive 1 gene, salicylic acid-dependent defense, Pseudomonas syringae, 789; Plum pox virus, multiple resistance traits, 541; Pseudomonas syringae, heterochromatin, hypomethylation and cytology, 577

Arredondo, F., 1302

Arroyo-Rodriguez, A. S., 1092

Asvarak, T., 139

Atanassova, R., 1103

Avocado, Dematophora root rot, biocontrol with bacteria, 418

Ayme, V., 557

Ayra, C., 399
Azarova, T., 250

Azocarcus spp.

-on rice, defense protein upregulation, jasmonate, 502

-on rice and Kallar grass roots, endoglucanase, 181

Bacteria

-type III secretion system, regulation, review, 1159

—virulence, HD-GYP domain, review, 1378

Badel, J. L., 99

Baehler, E., 313

Baillieul, F., 747

Baker, S. E., 139

Bakker, J., 521

Baldwin, T. K., 1444

Balogh, B., 69

Bamboo mosaic virus, satellite RNA-encoded P20, arginine-rich, cell-to-cell movement, 758

Banba, M., 80

Barajas, D., 904

Barley

-blast, resistance, relation to powdery mildew gene, 1034

-Fusarium graminearum, transcriptome analysis, 407

-Magnaporthe grisea, nonhost resistance, Pennisetum-infecting lineage, 1014

—powdery mildew, Mla resistance alleles, basal defense, 939

-rust, nonhost immunity, resistance gene sets, 1270,1472

Barny, M.-A., 16

Barsch, A., 998

Bashtovyy, D., 811

Battistoni, F., 502

Batut, J., 363

Baudouin, E., 970

Baum, T. J., 463

Beatty, M., 1240

Beckers, G. J. M., 1062

Beeckman, T., 1431

Beer, S. V., 53, 359

Belghazi, M., 502

Beliën, T., 1072

Bellizzi, M., 1216

Bemisia tabaci

-on eggplant, resistance, 383

—salicylic acid and resistance, kinase signaling, 655

Bender, C. L., 768

Bentéjac, M., 429

Bercovich, S., 682

Berger, S., 1138

Bergman, B., 1251

Bergström, E. T., 418

Besenyei, E., 161

Betti, M., 80

Bian, X.-Y., 614

Biligui, B., 550

Biological control

- fusaric acid for Orobanche ramosa, 550

-Pseudomonas fluorescens: for avocado root rot, 418; for Fusarium oxysporum on
ERRATA PAGE NUMBERS IN ITALICS

tomato, root exudate, 1121; root association, global regulators, 313

-Trichoderma virens, proteinaceous elicitor secretion, systemic resistance, 838

Blanchette, R. A., 150

Bloemberg, G. V., 418

Blumeria graminis, on barley:

-resistance alleles, basal defense suppression, 939

-resistance gene related to blast resistance, 1034

Bobik, C., 363

Boddu, J., 407

Bodogai, M., 811

Bogdanove, A. J., 240, 304

Bonasera, J. M., 53, 359

Boore, J. L., 1329

Borrás-Hidalgo, O., 399

Borroto, C. J., 399

Boscari, A., 896

Botrytis cinerea

- on Arabidopsis, salicylic acid and yeast effect, 1138

-on Arabidopsis thaliana, antisense and polygalacturonase-inhibiting protein, 931

- ethylene sensing and gene activation, fungusplant interaction, 33

-on grape: endopolygalacturonase elicited in defense, signaling network, 429; ergosterolinduced protection, stilbene synthase gene, 1103

—virulence, xylanase requirement, 25

— virulence factor, histidine kinase, 1042

Bouizgarne, B., 550

Boureau, T., 16

Bourque, S., 711

Bouteau, F., 550

Bozsó, Z., 161

Brachmann, A., 1082

Brassica napus, Leptosphaeria maculans, pathogenicity and ketoacyl thiolase, 588

Brisset, M.-N., 16

Brito, N., 25

Brome mosaic virus, gene silencing in monocotyledons, 1229

Bronstein, P. A., 1193

Brown, D., 1055

Bruand, C., 363

Buell, C. R., 1193

Buendía-Clavería, A. M., 43

Burnes, T. A., 150

Bursaphelenchus xylophilus, on pine, pectate lyases in esophageal gland, 280

Cafarchia, C., 1113

Cajanas cajan, nodulation, capsular polysaccharide role, 43

Cakir, C., 854

Caldelari, I., 200

Caldo, R. A., 939

Candresse, T., 541

Capela, D., 363

Caranta, C., 557

Cartinhour, S. W., 1151, 1167, 1193

Castillo, J. A., 69

Cazorla, F. M., 418 
Cecchini, N. M., 577

Cell death

-lettuce bacterial rot, 112

- Nicotiana benthamiana and tomato, synergism, 854

-Pantoea stewartii, on corn, 1092

Cervone, F., 931

Chacón, O., 399

Chadœuf, J., 557

Chagué, V., 33

Chaluvadi, S. R., 1229

Charkowski, A. O., 451

Charpentier, M., 80

Chen, J. C.-F., 758

Chen, W., 80, 758

Chen, Z. J., 257

Cho, B. H., 924

Cho, S., 407

Cho, Y., 7

Choi, Y.-E., 725

Chronis, D., 635

Cladosporium fulvum, on tomato:

—hydrolysis and plant chitinases, 1420

-hypersensitivity, genes, 567

Clifford, J. M., 69

Coba de la Peña, T., 173

Cochliobolus heterostrophus, T-toxin biosynthesis, genes required, 139

Collazo, C., 399

Colletotrichum spp., on Arabidopsis, nonhost resistance, actin filament function, 270

- C. graminicola, on maize, gene profiling, laser microdissection, 1240

Collmer, A., 99, 1151, 1167, 1180, 1193

Comai, L., 665

Conrath, U., 1062

Contributors, information, 92

Coplin, D. L., 1092

Coraiola, M., 1113

Corn (see Maize)

Cornelis, P., 1406

Couch, B. C., 288

Coughlan, S., 1240

Coutos-Thévenot, P., 1103

Cover photo

-Lotus japonicus: nitrogen-fixing root-nodule formation, October; root hair cells, calcium spike, August; spontaneous nodules, April

-Lycopersicon esculentum, stolbur phytoplasma, flowers, January

-onion, plasma membrane in epidermis, type III effector proteins, February

- Phytophthora ramorum and P. ramorum genome seqeunce, comparative analyses, December

-Plasmodiophora brassicae, root gall development, May

-Pseudomonas syringae, on cherry, plant signal molecules and toxins, March

- signal transduction, cryptogein-induced, schematic representation, July

-Tomato leaf curl virus, on tobacco, transgene expression, June

-wheat, abiotic and biotic stresses, hydrogen peroxide, September

-Xanthomonas campestris pv. campestris, on Florida mustard leaves, November

Cramer, R. A., Jr., 7

Crane, E., 1240

Cronartium ribicola, on pine, resistance proteins, proteomic comparison, 150

Crooks, C., 200

Crous, P. W., 1014

Cucumber mosaic virus, long-distance movement of particles, phloem protein 1 , 734

Culver, J. N., 864

Czelleng, A., 161
Dalla Serra, M., 1113

Damasceno, C. M. B., 1368

Dangott, L. J., 838

Danit, L.-V., 33

David, A. J., 150

Davis, E. L., 463

Davis, J. W., 7

Dean, R., 1055

Debellé, F., 495

de Bruijn, I., 699

Deckers, P., 1431

DeClerck, G. A., 1167, 1193

Decroocq, V., 541

de Felipe, M. R., 173

de Figueiredo, P., 665

de Graaff, L., 521

de Groot, P. W. J., 1348

de Jong, C. F., 567

de Kock, M. J. D., 699

de Koster, C. G., 1348

De Lorenzo, G., 931

Delrot, S., 1103

Delrow, J., 665

Dematophora necatrix, on avocado, biocontrol with Pseudomonas fluorescens, 2-hexyl 5propyl resorcinol correlation, 418

Deng, X., 976

Denny, T. P., 69

de Ruffray, P., 747

De Vleesschauwer, D., 1406

Devos, S., 1431

de Waard, M. A., 1262

de Werra, P., 313

de Wit, P. J. G. M., 567, 1420

Díaz-Camino, C., 1385

Díaz-Ruíz, J. R., 904

Ding, X. S., 1229

Ditt, R. F., 665

Dixelius, C., 958

Djonović, S., 838

Djordjevic, M. A., 215, 471

Dolan, M., 1216

Dong, R., 463

Dörner, L., 215

Dow, J. M., 1378

Downie, J. A., 914, 1456

Duan, Y. P., 69

Duckett, S. B., 418

Dumas-Gaudot, E., 988

Dusha, I., 811

Duvick, J., 1240

Ebbole, D., 1055

Eggenberger, A., 304

Ekman, M., 1251

El Hadrami, I., 550

Elliott, C. E., 588

El-Maarouf-Bouteau, H., 16, 550

Elphinstone, J., 69

Endoxylanases, microbial, use in plant defense system, review, 1072

Endophytes, bacterial, host interaction, review, 827

Engler, G., 970

Erhardt, M., 747

Erokhina, T. N., 874

Errata

-vol. 19, no. 1, 2006, 359

- vol. 19, no 9, 2006, 1291

-vol. 19, no. 11, 2006, 1472

Erwinia spp.

-E. amylovora: on apple, pathogenicity effector and proteins, interaction, 53, 359; on apple and tobacco, pathogenicity, type III effector, 16; on pear, virulence and Pseudomonas syringae relation, 644 -E. chrysanthemi: $d s p E$ and pelD regulation, population analysis, 451; pathogenesis, multidrug resistance systems, 607

Espino, J. J., 25

Essig, J. S., 123

Estrada-Navarrete, G., 1385

Ethylene

-maize defense response, insect herbivory, 189

- sensing, gene activation, Botrytis cinerea, 33

Eveillard, S., 62

Evidente, A., 1113

Eyquard, J. P., 541

Fan, Z. F., 948, 1291

Farman, M., 1055

Farmerie, W., 69

Ferenczi, S., 811

Ferrari, S., 931

Ferreira, A. O., 1167

Filipe, C., 363

Fillinger, S., 1042

Finley, L., 1359

Flores-Cruz, Z., 69

Flors, V., 1062

Fouhy, Y., 1378

François, J., 1103

Fray, R. G., 227

Fujikawa, T., 342

Fusarium spp.: on Arabidopsis thaliana, trichothecenes and elicitors, 512; fusaric acid, control for Orobanche ramosa, 550

$-F$. graminearum: on barley, transcriptome analysis, 407; virulence and female fertility, FSR 1, 725; on wheat, resistance engineered, Arabidopsis NPR1 expression, 123

$-F$. oxysporum, on tomato, biocontrol by Pseudomonas fluorescens, tomato root exudates, 1121

$-F$. verticillioides, virulence and female fertility, FSR1, 725

Gabriel, D. W., 69

Gabriëls, S. H. E. J., 567

Galletti, R., 931

García, J. A., 350, 541

García-Agustín, P., 1062

García-Arenal, F., 734

Garcia-Brugger, A., 711

Garnier, A., 16

Geil, R., 1082

Gemmer, S., 181, 502

Genomes

-Phytophthora spp.: decoded, 1295; phospholipid signaling genes, 1337

-Phytophthora sojae and P. ramorum, mitochondrial DNA, variation, 1329

- secretomes, plant defense and pathogenicity, 1368

Genes

- AL2 and AL3, Tomato golden mosaic virus, sequence elements, 1394

- coregulated, Pseudomonas syringae on tomato and Arabidopsis, profiling, 1167

-effector, Xanthomonas spp., defense responses, 342

-hemoglobin, Alnus firma, nitric oxide detoxification, 441

-IrpE and Ralstonia solanacearum, Hrp pili production and virulence, 884

- jasmonate-insensitive 1, Arabidopsis thaliana, 789

—nodD and nodRL, Rhizobium leguminosarum, LCOs, 215

-parasitism, root-knot nematodes, encoded proteins, 463

-Phytophthora spp., protein secretion, synteny, 1311

-Rmo1, barley blast, relation to Mla, 1034

- tobacco leucine-rich repeat, modulator in hypersensitivity, 747 
Geranium, Ralstonia solanacearum, race 3 , biovar 2, identification, 69

Ghannam, A., 747

Gianinazzi, S., 988

Gijzen, M., 1295

Gilman, J. H., 150

Gil-Serrano, A. M., 43

Giovanini, M. P., 1023

Gobena, D., 1359

Godfroy, O., 495

Goggin, F. L., 383

Gomès, E., 1103

González, C., 25

González, E. T., 69

González-Sama, A., 173

Goodin, M. M., 1207

Gordon, J. S., 1167

Govers, F., 1348, 1337, 1311, 1295

Goverse, A., 521

Grape, Botrytis cinerea:

-endopolygalacturonase elicited in defense, signaling network, 429

- ergosterol-induced protection, stilbene synthase gene, 1103

Greenberg, J. T., 69

Gresshoff, P. M., 80, 1385

Groenewald, J. Z., 1014

Gross, D. C., 257

Guillén, G., 1385

Gutiérrez, R., 43

Guttman, D. S., 1193

Hakoyama, T., 80

Haller, K., 1055

Ham, J. H., 1092

Hamdi, S., 1103

Hammond-Kosack, K. E., 1444

Han, S. H., 924

Harada, K., 80

Harel, A., 682

Harfouche, A. L., 189

Harrison, S. J., 1420

Harteveld, M., 215

Hata, S., 80

Hayashi, M., 80, 801, 1456

Hayes, P. M., 1034

He, S.-Y., 644

Hebert, S., 383

Heckmann, A. B., 1456

Held, M., 1082

Helder, J., 521

Hernould, M., 62

Hibiscus chlorotic ringspot virus, p27 and isoforms, movement in kenaf, 948, 1291

Hidalgo, Á., 43

Higashi, K., 801

Higashi, S., 441

Higgins, L., 150

Hikichi, Y., 112

Hill, J., 304

Hio, T., 801

Höfte, M., 1406

Hori, K., 1034

Hossain, Md. S., 80, 780

Howell, C. R., 838

Howlett, B. J., 588

Hsu, Y.-H., 758

Huang, G., 463

Huang, Q., 69

Huitema, E., 854

Hurek, T., 181

Hurtado-Gonzales, O., 1359

Hussey, R. S., 463

Hypersensitivity

- tobacco leucine-rich repeat gene, modulator, 747

-on tomato, gene identification, Cladosporium fulvum, 567
Iacobellis, N. S., 1113

Ichimura, K., 512

Imaizumi-Anraku, H., 80, 801

Inderbitzin, P., 139

Inukai, T., 1034

Inzé, D., 1431

Ishihara, H., 342

Jacobs, J. J., 150

Jacquemond, M., 557

Jacques, A., 747

Jafary, H., 1270, 1472

Jakab, G., 1062

James, E. K., 373

Jarosch, B., 1014

Jia, L., 383

Jiang, R. H. Y., 1311, 1302

Jiménez, I., 350

Joardar, V., 789, 1193

Johansson, A., 958

Jones, J. (Fla.), 69

Jones, J. T. (U.K.), 280

Joosten, M. H. A. J., 567, 1420

Jordan, K. L., 1180

Kaloshian, I., 655

Kamilova, F., 250, 1121

Kamoun, S., 854

Kanamori, N., 80

Kanneganti, T.-D., 854

Karas, B., 80, 1082

Kasiviswanathan, V., 758

Kato, T., 80

Kauffmann, S., 747

Kawaguchi, M., 80, 801

Kawasaki, S., 80

Keel, C., 313

Keen, N. T., 451

Keller, I., 480

Kelley, B. S., 1368

Kellmann, J.-W., 874

Kema, G. H. J., 389, 1262

Kenerley, C. M., 838

Kerr, K. F., 665

Kevei, Z., 173

Kiba, A., 112

Kikuchi, T., 280

Kim, B.-D., 1368

Kim, B.-S., 1368

Kim, J. F., 53, 359

Kim, K. Y., 924

Kim, K.-H., 7

Kim, Y. C., 924

Kim, Y. W., 924

Kimura, M., 512

Klement, É., 161

Klement, Z., 161

Klint, J., 1251

Klis, F. M., 1348

Ko, A., 1302

Kodama, M., 1280

Koehler, J., 1444

Kojima, T., 80

Kondorosi, E., 173

Kouchi, H., 80, 780

Krampis, K., 1329

Kravchenko, L. V., 250, 1121

Krishnan, H. B., 635

Kroken, S., 139

Kruger, W. M., 407

Krusell, L., 80

Kumagai, H., 80

Kunkel, B. N., 768, 789

Kunkuru, A. R., 1042

Kunz, S., 480

Küster, H., 998

Lamboy, W. F., 1167

Lamotte, O., 711
Lamour, K. H., 1359

Lan, L., 976

Lansac, M., 541

Laquitaine, L., 1103

Larsen, K., 80

Laukens, K., 1431

Laurie-Berry, N., 789

Lawrence, C. B., 7

Lazzaroni, S., 1113

Le, P. T., 227

Leach, J. E., 342

Lechner, M., 625

Lecourieux, D., 711

Lee, M. C., 924

Lee, M.-K., 1302

Lee, S. J., 924, 1368

Lee, Y.-W., 725

Le Gall, O., 541

Legendre, L., 1042

Lei, W., 43

Le Pêcheur, P., 1042

Leptosphaeria maculans, on Brassica napus, pathogenicity, ketoacyl thiolase, 588

Leroux, P., 1042

Le Rudulier, D., 896

Lesemann, D.-E., 874

Lettuce, bacterial rot, apoptotic cell death, 112

Li, B.-Y., 139

Li, D., 1385

Li, H. F., 948, 1291

Li, Q., 655

Lin, N.-S., 758

Lindeberg, M., 1151, 1193

Lipka, V., 270

Liu, G., 1216

Liu, L., 69

Liu, Q., 567

Liu, W., 1042

Lo Cantore, P., 1113

Lombardo, F., 1456

López, L., 350

López, Y., 399

López-Baena, F. J., 43

López-Solanilla, E., 607

Lorio, J. C., 635

Lotus japonica

—defective mutants, identification, 1456

-hypernodulation phenotype, genetic suppressors, 1082

-infection thread development, nodulation mutants, 801

—nodule organogenesis, endoreduplication, mitotic inhibitor, 173

—root hair cells, calcium spike, cover photo, August

—root hairs, calcium signaling, Nod factor, 914

-root-nodule formation: nitrogen fixing, mutant, cover photo, October; nodulation without rhizobia, 373

-spontaneous nodules, cross between lines, cover photo, April

-symbiosis genetics, maps and loci, 80

- symbiotic mutant, nodule infected cells, symbiosomes, 780

Lu, G., 1216

Lu, S.-E., 257

Lucas, M. M., 173

Lucey, J. F., 1378

Ludwig-Müller, J., 480

Lugtenberg, B. J. J., 250, 418, 1121

Luthe, D. S., 189

Lycopersicon esculentum (see also Tomato)

-stolbur phytoplasma, flower infections, cover photo, January

MacGillivary, M., 1082

Mackey, D. M., 1092

Macrosiphum euphorbiae 
-on eggplant, resistance, 383

-on potato, tomato resistance gene, salicylic acid, 655

Madiona, K., 550

Madsen, E., 80

Madsen, L. H., 80

Maes, T., 181

Maggiorani Valecillos, A., 607

Magnaporthe spp.

-M. grisea: on barley, nonhost resistance, 1014; on rice, jasmonic acid effect, rice allene oxide synthase gene, 1127 ; on rice,

MAP kinase gene family, molecular analysis, 530; on rice, $\mathrm{Pi} 2$ and $\mathrm{Piz}-\mathrm{t}$ resistance proteins, 1216; on rice, web-based database, study resource, 1055

-M. oryzae, on barley, resistance, relation to Maize powdery mildew resistance gene, 1034

-anthracnose stalk rot, gene profiling and laser microdissection, 1240

- defense response to insect herbivory, ethylene signaling, 189

- Pantoea stewartii, cell death and effector protein, 1092

Majerczak, D. R., 1092

Makandar, R., 123

Makarova, N., 250, 1121

Makino, S., 240

Mandon, K., 896

Mann, S., 200

Marchive, C., 1103

Margaret, I., 43

Marquez, A. J., 80

Martin, G. B., 1167

Martínez-Romero, E., 827

Masuda, D., 512

Mathys, S., 313

Mauch, F., 1062

Mauch-Mani, B., 1062

Maurhofer, M., 313

May, G., 288

Mayama, S., 112

Mayetiola destructor, on wheat, gene-for-gene defense, 1023

Medicago spp.

- M. sativa, bacteroids, betaine transporter and nodulation, salt stress, 896

-M. truncatula: arbuscular mycorrhiza, root proteome, mutations, 988; protein kinase, nodulation restoration, 495; Sinorhizobium meliloti, nitric oxide formation, 970 ; symbiotic nitrogen fixation, 330

Medzihradszky, K. F, 161

Mehrabi, R., 389, 1262

Mei, C., 1127

Meijer, H. J. G., 1359, 1348, 1337

Meloidogyne spp.: parasitism genes, plant transcription factor, 463; resistance from tomato $\mathrm{Mi}$ - 1 gene, 655

-M. incognita, endo-xylanase from, symbiontindependent, 521

-M. javanica, on eggplant, tomato gene confers resistance, 383

Mendgen, K., 625

Meng, X., 53, 359

Mergaert, P., 173

Mesorhizobium loti, on Lotus japonicus, hypernodulation phenotype and genetic suppressors, 1082

Miché, L., 502

Miclea, P., 811

Mikhailova, N., 69

Minina, E. A., 874

Mitchell, T., 1055

Mitreva-Dautova, M., 521

Mittapalli, O., 1023

Miwa, H., 914, 1456
Möll, U., 625

Monestiez, M., 550

Moon, J. H., 924

Moreno, J., 43

Moreno-Hagelsieb, G., 1167

Morozov, S. Y., 874

Morris, A. C., 471

Mouras, A., 62

Moury, B., 557

Muehlbauer, G. J., 407

Mueller, M. J., 1138

Mukaihara, T., 884

Mulder, L., 80

Mulholland, V., 69

Müller, J., 80

Murakami, Y., 80

Murata, Y., 884

Murillo, E., 1385

Murooka, Y., 801

Murray, J., 80, 1082

Mushrooms, Pseudomonas reactans, white lineinducing principle, 1113

Mycorrhizae, arbuscular, Medicago truncatula, root proteome, 988

Mycosphaerella graminicola, on wheat: dimorphism and pathogenicity, $\mathrm{MgHog} 1$ regulation, 1262; MAP kinase gene, role in penetration and growth, 389

Mycotoxin, T-toxin, Cochliobolus heterostrophus on corn, biosynthesis, 139

Myers, C. R., 1151, 1167, 1193

Nagel, W., 480

Nakaho, K., 884

Nakashita, H., 512

Nakayashiki, H., 112

Navarre, D. A., 655

Nelson, R. S., 1229

Nemacheck, J. A., 1023

Nester, E. W., 665

Nettleton, D., 939

Newman, M.-A., 1062

Nicotiana benthamiana (see also Tobacco)

-Bamboo mosaic virus, satellite RNA-encoded P20, 758

— cell death, synergism, Phytophthora infestans, 854

- Phytophthora infestans, hypersensitivity genes, 567

-Plum pox virus, CI-interacting protein, 350

Niehaus, K., 998

Niks, R. E., 1270, 1472

Nishiuchi, T., 512

Nissinen, R. M., 53, 359

Nitrogen fixation

—Lotus japonicus, symbiotic mutant, symbiosomes, 780

-Sinorhizobium meliloti on Medicago truncatula, symbiosis, 330

Nodulation

—alfalfa, metabolite profiles, physiological adaptations, 998

-Lotus japonicus: infection thread, 801; Mesorhizobium loti, genetic suppressors, 1082; nodules without rhizobia, 373; root hairs, calcium signaling, 914

-Medicago truncatula-Sinorhizobium meliloti, nitric oxide formation, 970

-restoration in Medicago truncatula, protein kinase, 495

—Sinorhizobium meliloti: on alfalfa, transcriptomic dissection, 363; on Medicago sativa, betaine transporter and bacteroids, 896

- soybean and Cajanas cajan, capsular polysaccharide role, 43

Noreen, S., 418

Norman, D., 69

Nostoc sp., endosymbiotic, profile and proteomic approach, 1251

Nowakowski, K., 1082

O’Connell, R., 270

Odijk, R., 418

Oh, H.-S., 99

Ohm, H. W., 1023

Ohnishi, K., 112

Ohtomo, R., 80

Okuno, T., 270

Oldroyd, G. E. D., 914

Olivares, J.-E., 1385

Ollero, F. J., 43

Olsthoorn, M. M. A., 215

Onion, epidermal cell, plasma membrane, type

III effector proteins, cover photo, February

Ooki, Y., 80

Orbach, M. J., 1055

Orobanche ramosa, biocontrol with fusaric acid, 550

Oryza sativa (see also Rice)

-Azocarcus endophyte, jasmonate-inducible defense proteins, upregulation, 502

Otani, H., 1280

Ott, P. G., 161

Ouhdouch, Y., 550

Overmars, H., 521

Paape, M., 874

Padmanabhan, M. S., 864

Palenzuela, P. R., 607

Palloix, A., 557

Palmer, T., 200

Pampanwar, V., 1055

Pantoea stewartii, on corn, cell death and effector protein, 1092

Papp, P., 811

Parada, M., 43

Park, K. H., 924

Park, P., 112

Parniske, M., 80, 480, 1082, 1456

Pascal, S., 1103

Pathogenicity, pathogen-host interactions database, 1444

Pathosystem, Arabidopsis-Verticillium longisporum, signals, 958

Patnaikuni, M., 69

Patschkowski, T., 998

Pauly, N., 970

Pavet, V., 577

Pear, fire blight, virulence gene, Pseudomonas syringae relation, 644

Péchy-Tarr, M., 313

Penaloza-Vazquez, A., 768

Peng, J., 939

Peng, Q., 451

Pennarun, A. M., 550

Pennisetum sp., Magnaporthe grisea on barley, relation, nonhost resistance, 1014

Perino, C., 16

Peronospora hyoscyami, on tobacco, transcription factor and glutathione synthetase, defense requirement, 399

Perry, J., 80, 1082, 1456

Peters, J., 567

Petersen, T. R., 80

Peterson, L., 1082

Petunia, Ralstonia solanacearum, lateral root formation, Hrp mutants, 597

Phaseolus spp. =Agrobacterium rhizogenes, transformation, functional genomics, 1385

-P. vulgaris, halo blight, HrpL regulon and type III secretion system effector proteins, 1193

Phylogeny, Solanum spp., homolog evolution, 288

Phytophthora spp.: gene mutation, targeted, 1359; genomes compared, mitochondrial 
DNA, 1329; genomes, RNA gene transfer, codon usage, 1322; genomics, decoded, 1295; phospholipid signaling genes, genome analysis, 1337; protein secretion, synteny and gene duplication and deletion, 1311

$-P$. infestans: cell death pathways, synergistic interactions, 854; on Nicotiana

benthamiana, hypersensitivity genes, 567 on tomato, secretomes, characterization screen, 1368

$-P$. ramorum, proteins, cell wall association 1348; genome sequence, cover image, December

$-P$. sojae, on soybean, genome sequence map, bacterial artificial chromosome-based, 1302 genome sequence, cover image, December

Phytoplasma, stolbur, on tomato:

- cover photo, January

-flower abnormality, 62

Phytotoxin, trichothecenes, Fusarium on

Arabidopsis, elicitor similarity, 512

Pieterse, C. M. J., 1062

Pieuchot, L., 970

Pike, J., 80

Pinus strobus, blister rust, resistance proteins, 150

Plant defense system, microbial endoxylanases, breaching the barrier, review, 1072

Plasmodiophora brassicae

-on Arabidopsis: initial metabolism, proteome and hormone, 1431; transcriptome analysis, cytokinins, 480

-root gall development, Arabidopsis, cover photo, May

Plum pox virus

-on Arabidopsis thaliana, multiple resistance traits, 541

-CI-interacting protein, 350

Poinssot, B., 429, 711, 1062

Polepalli, J. S., 1042

Portieles, R., 399

Potato virus $X$, chimeric virus infection, RNA silencing, 904

Potato virus $Y$, on pepper, resistance genes, 557

Pozo, M. J., 838, 1062

Pracros, P., 62

Presting, G., 69

Priming, plant infection, induction of physiological state, review, 1062

Prinsen, E., 143

Pseudomonas spp., cyclic lipopeptide production, biosynthesis, review, 699

-P. aeruginosa: Magnaporthe grisea affected by, Rhizoctonia solani relation, 1406; on tobacco, $N$-acyl-homoserine lactone synthase genes, 227

-P. chlororaphis, systemic resistance determinant, tobacco, 924

$-P$. cichorii, on lettuce, bacterial rot and apoptotic cell death, 112

-P. fluorescens: biocontrol agent, global regulators, 313; biocontrol of avocado root rot, 418; biocontrol of tomato foot and root rot, root exudate effect, 1121

$-P$. reactans, on cultivated mushrooms, white line-inducing principle, 1113

-P syringae: on Arabidopsis, heterochromatin, 577; on Arabidopsis, salicylic acid, jasmonate-insensitive 1 gene, 789; on Arabidopsis, salicylic acid and yeast effect, 1138; on bean, HrpL regulon and type III secretion system effector proteins, 1193; on cherry, toxins and plant signal molecules, cover photo, March; lipodepsipeptide phytotoxins, genes encoding, plant signal molecules, 257; model strains, genes encoding regulon and secretion system effectors, review, 1151 on pear, fire blight affected by, virulence gene, 644; on soybean, cultivar-specific avirulence, transgene expression, 304; on tobacco, basal resistance, bacteria as markers, elicitors, 161; on tomato, avirulence and virulence, type III effector, 130; on tomato, multiple virulence components, CorR regulation, 768; on tomato, symptom reduction by mutant, 99 ; on tomato, Tat pathway, optimal virulence, 200; on tomato and Arabidopsis, pathway regulation, $h r p L$ and $h r p R S$, 976; on tomato and Arabidopsis, profiling defines $\mathrm{HrpL}$ regulon, coregulated genes, 1167; on tomato and Arabidopsis, type III secretion system effector proteins, 1180

Puccinia spp., on barley, resistance genes and nonhost immunity, 1270, 1472

Pucheu, I., 16

Pueyo, J. J., 173

Pugin, A., 429, 711, 1062

Pujol, M., 399

Puppo, A., 970

Puthoff, D. P., 1023

Qi, M., 1127

Qu, S., 1216

Quintero, C., 577

Quinto, C., 1385

Raacke, I. C., 1138

Raaijmakers, J. M., 699

Radu, T. G., 80

Radutoiu, S., 80

Ralstonia solanacearum

-on petunia, lateral root formation, Hrp mutants, 597

— pili production and virulence, $\operatorname{IrpE}$ gene and Hrp, 884

-race 3 biovar 2, open reading frame identification, 69

Ramos, C., 288

Rasheed, M. S., 614

Rawlings, C., 1444

Recorbet, G., 988

Reddy, J., 69

Reinhold-Hurek, B, 181, 502

Renaudin, J., 62

Requena, A., 734

Resistance

-Arabidopsis to Plum pox virus, multiple traits, 541

-barley, nonhost, Magnaporthe grisea, 1014

-Capsicum spp. to Potato virus $Y, p v r 2^{3}$ gene, 557

- Pinus strobus to Cronartium ribicola, proteomic comparison of needles, 150

- R genes, diversity in Solanum spp., evolution, 288

—rice to: blast, jasmonic acid, 1127; blast, $\mathrm{Pi} 2$ and Piz-t resistance proteins, 1216 Magnaporthe grisea, factors, 1406; Xanthomonas oryzae, resistance genemediated defense, 240

- system, tobacco, Erwinia and Pseudomonas spp. comparison, butanediol as determinant, 924

— systemic, Trichoderma virens effect, 838

- tobacco to blue mold, transcription factor and glutathione synthetase, 399

-wheat to Hessian fly, gene-for-gene defense, 1023

Revers, F., 541

Reviewers, acknowledgment, 3

Reyna, N. S., 530

Rezaian, M. A., 614

Rhizobium leguminosarum

—nodD and nodRL genes, LCOs regulated by, 215
- on subterranean clover, nodule and lateral root-generating signals, 471

Rhizosphere, vegetable exudates, bacteria affected by, 250

Rice (see also Oryza sativa)

-blast: jasmonic acid effect, allene oxide synthase gene, resistance, 1127; Pi2 and Pizt resistance proteins, 1216; web-based database for studying, 1055

-Magnaporthe grisea: MAP kinase gene family, molecular analysis, 530; Rhizoctonia solani, Pseudomonas aeruginosa effect, 1406

-root endophyte, endoglucanase and infection, 181

-Xanthomonas oryzae, resistance genemediated defense, 240

Rispail, N., 80

Robben, J., 1072

Robert, F., 988

Robert-Seilaniantz, A., 130

Rodríguez-Carvajal, M. A., 43

Rona, J. P., 550

Rosa, A. L., 577

Rose, J. K. C., 1368

Rosenberg, C., 495

Rosenblueth, M., 827

Ross, L., 1082

Rouf Mian, M. A., 1229

Roze, E., 521

Ruiz-Sainz, J. E., 43

Ryan, R. P., 1378

Saddler, G., 69

Sagaram, U. S., 725

Saito, K., 80

Sakai, H., 1216

Salcedo, G., 734

Salicylic acid, tomato, aphid resistance, kinase signaling, 655

Salt stress, adaptation, Medicago sativa nodulation, 896

Saltzmann, K. D., 1023

Samac, D. A., 330

Sánchez, F., 1385

Sánchez-Guevara, N., 1385

Sandal, N., 80, 373

Sangawa, Y., 112

Santana, O., 1385

Santos, F. A., 1302

Sarx, J., 480

Sasakura, F., 441

Sato, K., 1034

Sato, S., 80

Savka, M. A., 227

Schaffrath, U., 1014, 1062

Schapaugh, M. A., 123

Schechter, L. M., 1151, 1180

Schell, M., 69

Schepetilnikov, M. V., 874

Scheuring, C., 1302

Schiemann, J., 874

Schlaman, H. R. M., 215

Schmülling, T., 480

Schneider, D. J., 1151, 1167, 1180, 1193

Schneider, S. E., 1180

Schneider, W. L., 1229

Schots, A., 521

Schuller, A., 480

Schulze Gronover, C., 33

Schulze-Lefert, P., 270

Sclerotinia sclerotiorum, sclerotial development pathogenicity, calcineurin requirement, 682

Scott, R. A., 227

Seemanpillai, M. J., 614

Shah, G., 383

Shah, J., 123

Shan, L., 130 
Shaposhnikov, A. I., 250, 1121

Sharon, A., 33

Sheng, G., 1127

Shi, L., 139

Shibata, S., 80

Shibuya, H., 280

Shiferaw, H., 864

Shim, W.-B., 725

Shimada, C., 270

Shimizu, R., 99

Shimoda, Y., 441

Shinozaki, K., 512

Shivaji, R., 189

Shukle, R. H., 1023

Shung, C.-Y., 1394

Sicard, O., 541

Siemens, J., 480

Siewers, V., 33

Signal transduction, cryptogein-induced, schematic representation, cover photo, July

Signaling events, plant defense elicitors, review, 711

Silencing, Tomato leaf curl virus, DNA methylation, 614

Simón-Buela, L., 734

Sinorhizobium spp.

-S. fredii: cysteine synthase encoded, open reading frame, 635; on soybean and Cajanas cajan, nodular, capsular polysaccharide, 43

-S. meliloti: on alfalfa, symbiosis, transcriptomic dissection, 363; ntrPR operon, toxin-antitoxin module, 811; on Medicago sativa, bacteroids, nitrogen fixation, 896; on Medicago truncatula, nitric oxide formation, 970; on Medicago truncatula, nitrogen fixation, 330

Sirasanagandla, S. S., 1394

Skøt, L., 80

Smant, G., 52

Smith, J. A., 150

Smith-Becker, J., 655

So, J., 725

Soderlund, C., 1055

Solanum spp., resistance genes, R genes, 288

$-S$. melongena, nematode and aphid resistance, tomato gene, 383

Solovyev, A. G., 874

Souche, S., 557

Soybean

- cultivar-specific avirulence, Soybean mosaic virus and Pseudomonas syringae, 304

—nodulation, capsular polysaccharide role, 43

-Phytophthora sojae, genome sequence map, integrated bacterial artificial chromosome, 1302

-Sinorhizobium fredii, cysteine synthase encoded, open reading frame, 635

Soybean mosaic virus, avirulence adapted for transgene expression, 304

Spaink, H. P., 215

Spangler, R., 288

Spodoptera frugiperda, on maize, defense response and ethylene signaling, 189

Sreedharan, A., 768

Staal, J., 958

Stavrinides, J., 1193

Steeber, D. A., 451

St. John, B., 1368

Stocker, R., 189

Stougaard, J., 80, 373, 1082

Street, I. H., 789

Suganuma, N., 80

Sugio, A., 240

Sun, J., 914

Sun, Q.-H., 7

Sundin, G. W., 644

Sunter, G., 1394

Sunter, J., 1394
Suzuki, A., 441

Swanson, J., 69

Szabo, L. J., 1270, 1472

Szatmári, Á., 161

Szczyglowski, K., 80, 1082

Tabata, S., 80

Takano, Y., 270

Takenouchi, K., 441

Takken, F. L. W., 567

Tamura, N., 884

Tang, W., 1240

Tang, X., 130, 976, 1159

Tansengco, M. L., 801

Tellström, V., 998

Tenllado, F., 904

Tesfaye, M., 330

Thomas-Oates, J. E., 215, 418

Thomma, B. P. H. J., 399

Tian, F., 1193

Tian, S. K., 1302

Tierney, M., 1359

Timmers, T., 495

Tirichine, L., 373

Tobacco (see also Nicotiana benthamiana)

-blue mold, transcription factor and glutathione synthetase, defense requirement, 399

-hypersensitivity, in NtLRP1 gene and as modulator, 747

$-N$-acyl-homoserine lactone synthase genes, Pseudomonas aeruginosa and Yersinia enterocolitica, 227

- nonhost for Erwinia amylovora, type III effector, 16

-Pseudomonas syringae, bacteria as resistance markers, elicitors, 161

-systemic resistance, determination, Erwinia compared to Pseudomonas, 924

Tobacco mosaic virus, replicase protein, Aux/AA proteins, 864

Tollbäck, P., 1251

Tomato (see also Lycopersicon esculentum

-bacterial speck, type III effector, avirulence and virulence, 130

— cell death, synergism, Phytophthora infestans, 854

-Cladosporium fulvum, hypersensitivity response genes, 567

-flower abnormality, stolbur phytoplasma, 62

- foot and root rot, biocontrol with Pseudomonas fluorescens, root exudate effect, 1121

-leaf mold, hydrolysis and plant chitinases, 1420

- Mi-1 gene, resistance and salicylic acid, kinase signaling, 655

- Mi-1.2 gene confers resistance to eggplant, 383

- Pseudomonas syringae: lesions reduced by mutant, 99; optimal virulence, Tat pathway, 200; pathway regulation by $h r p L$ and $h r p R S$ 976; profiling, HrpL regulon, coregulated genes, 1167; type III secretion system effector proteins, 1180

Tomato golden mosaic virus, viral sequence elements, gene expression, 1394

Tomato leaf curl virus

—-silencing escape, analysis, 614

-on tobacco, transgene expression, cover photo, June

Tomato spotted wilt virus, movement protein interactor, new protein family, 874

Ton, J., 1062

Tosa, Y., 112

Transcriptome analysis

-Arabidopsis thaliana and Agrobacterium tumefaciens, 665
-barley-Fusarium graminearum interaction, 407

—clubroot of Arabidopsis, cytokinins, 480

Trichoderma virens, proteinaceous elicitor secreted from, systemic resistance, 838

Trick, H. N., 123

Trifolium subterraneum, root nodule development, signals, 471

Tripathy, S., 1302, 1322

Tsuyumu, S., 342

Tuck, E., 80

Tudzynski, B., 33

Tudzynski, P., 33

Turgeon, B. G., 139

Turk, S. C. H. J., 567

Tyler, B. M., 1302, 1311, 1329, 1322

Uchiumi, T., 441

Ullman, D. E., 383

Umehara, Y., 80, 780

Urban, M., 1444

Uromyces fabae, on Vicia faba, invertase, infection course, 625

Vairo, D., 931

Vales, M. I., 1034

Valli, A., 350

Van Campenhout, S., 1072

Vance, C. P., 330

Vandelle, E., 429, 711

van den Burg, H. A., 1420

van der Lee, T., 389

Van Der Straeten, D., 1431

Van de Sype, G., 896

van de Vondervoort, P. J. I., 1348

Van Gijsegem, F., 597

Van Montagu, M., 181

Van Onckelen, H., 1431

Varga, G. J., 161

Vegetables, exudates, effect on stonewool and rhizosphere bacteria, 250

Vencato, M., 1167, 1180, 1193

Verticillium longisporum, on Arabidopsis, pathosystem, cytosolic NPT 1 and RFO1, 958

Vervoort, J., 1420

Viaud, M., 1042

Vicia faba, rust, invertase, infection course, 625

Vickers, K., 80

Vijaya Palani, P., 758

Vinardell, J. M., 43

Viruses, plant response elucidated, global impact, review, 1207

Voegele, R. T., 625

Volckaert, G., 1072

von Bodman, S. B., 227

von Rad, U., 1138

Vossen, J. H., 567

Waalwijk, C., 389

Wachowski, L. K., 567

Wagg, C., 1082

Walunas, T., 69

Wang, G.-L., 1055, 1216

Wang, J., 7, 257

Wang, L., 304

Wang, N., 257

Wang, T. L., 80, 1456

Wang, X., 80

Webb, J., 80

Wehling, M. D., 1167

Weil, J., 227

Wendehenne, D., 429, 711, 1062

Wheat

- abiotic and biotic stresses, hydrogen peroxide, cover photo, September

-Fusarium head blight, engineered resistance, Arabidopsis NPR1, 123 
-Hessian fly, gene-for-gene defense, oxidative burst, 1023

- Mycosphaerella graminicola, MAP kinase gene, penetration and growth role, 389

- Septoria tritici leaf blotch, dimorphism and pathogenicity, $\mathrm{MgHog} 1$ regulation, 1262

White, F., 240

Whitham, S. A., 1207

Wick, L. Y., 313

Wilkinson, H. H., 725

Williams, C. E., 1023

Williams, P. (U.K.), 227

Williams, P. W. (Miss.), 189

Williamson, V. M., 383

Wing, R., 1055

Winnenburg, R., 1444

Wirsel, S., 625

Wise, R. P., 939

Witsenboer, H. M. A., 567

Witters, E., 1431

Witthuhn, B. A., 150

Wong, S. M., 948, 1291
Wu, C., 1302

Xanthomonas spp., defense responses, avrBs3/pthA gene family, 342

-X. campestris: HD-GYP domain,virulence, review, 1378

-X. campestris pv, campestris, cover photo, November

-X. oryzae, on rice, resistance gene-mediated defense, inhibition, 240

Xiao, Y., 1159

Xie, Q.-G., 655

$\mathrm{Xu}$, J.-R. (Indiana), 1055

$\mathrm{Xu}, \mathrm{Z} ., 1302$

Yamagishi, D., 1280

Yamaguchi, I., 512

Yamaguchi, K., 512

Yang, C. (Iowa), 1207

Yang, C.-H. (Wis.), 451

Yang, K. Y., 924

Yang, S., 451

Yang, Y., 530, 1127

Yano, K., 80, 801, 1456

Yao, J., 69
Yao, N., 112

Yap, M.-N., 451

Yarden, O., 682

Yersinia enterocolitica, on tobacco, $N$-acylhomoserine lactone synthase genes, 227

Yin, Q. Y., 1348

Yoder, O. C., 139

Yokota, K., 80

Yoshida, S., 80, 512

Yoshikawa, M., 80

Zellerhoff, N., 1014

Zhang, H.-B., 1302

Zhang, X., 1302

Zhao, Y., 644

Zhou, B., 1216

Zhou, J., 976

Zhou, J.-M., 130, 1159

Zhou, T., 948, 1291

Zhukov, A., 69

Zimmerli, L., 1062

Zolobowska, L., 597

Zwiers, L.-H., 1262 